Philosophical Magazine B, 1998, Vol. 78, Nos. 5/6, 497-501

\title{
Theoretical investigations of spin-resolved appearance potential spectroscopy for $\mathrm{Fe} / \mathrm{Cu}(001)$ overlayers
}

\author{
V. Popescu, H. Ebert \\ Institut für Physikalische Chemie, Universität München, Theresienstr. 37, \\ D-80333 Munich, Germany \\ L. Szunyogh†, U. Pustogowa
}

Center of Computational Materials Science, TU Vienna, Austria and

$\dagger$ Department of Theoretical Physics, TU Budapest, Hungary

and P. Weinberger

Institut für Technische Elektrochemie, and Center of Computational Materials Science, TU Vienna, Austria

\begin{abstract}
We present spin-resolved appearance potential spectroscopy (APS) calculations at the $2 \mathrm{p}_{3 / 2}$ edge of $\mathrm{Fe}$ in the high-moment ferromagnetic phase of fcc $\mathrm{Fe} / \mathrm{Cu}(001)$ overlayers, based on a single-particle description of the underlying electronic structure. Within the framework of the real-space Korringa-KohnRostoker method, the spectra were calculated in a layer-resolved mode. This allows one to investigate in detail the dependence of the total APS signal - a superposition of weighted layer-signals - as a function of the Fe overlayer thickness.
\end{abstract}

\section{$\S 1$. Introduction}

During the last few years, the $\mathrm{Fe} / \mathrm{Cu}(001)$ system has attracted much attention because of its rich structural and magnetic properties (Thomassen et al. 1992, Li et al. 1994, Detzel et al. 1995). Due to the small epitaxial misfit, a good layer-by-layer growth of $\mathrm{Fe}$ on $\mathrm{Cu}(001)$ was expected and-for room-temperature grown samples - experimentally found, stabilizing the film in a fcc phase up to about 10 monolayers (Detzel et al. 1996). The magnetic structure of these samples depends strongly on the atomic volume and, at the lattice constant of $\mathrm{Cu}$, the system is at the edge of three different phases: a high-moment ferromagnetic, a low-moment ferromagnetic and a low-moment antiferromagnetic phase. The present paper continues a previous investigation (Ujfalussy et al. 1996) on the ferromagnetic state.

The spin-resolved appearance potential spectroscopy (APS), which is essentially an inverse core-valence-valence (CVV) Auger emission, was proved to be a powerful technique to investigate surface, interface and thin-film magnetism (Kirschner 1984, Ertl et al. 1993, Detzel et al. 1995). This core-level spectroscopy is element specific and, owing to the mean free path of the incoming electrons, the signal is not affected by that of the substrate.

A theoretical approach of the APS on the basis of a single-particle description of the underlying electronic structure was proposed recently (Ebert and Popescu 1997) and successfully applied to pure elements as well as compounds (Reinmuth et al. 
1997). This approach, used in a layer-resolved mode, is applied here to the ferromagnetically ordered system $\mathrm{Fe}_{n} / \mathrm{Cu}(001)$, with $n=1, \ldots, 6$.

\section{§2. Method of calculations}

The work presented here was performed on the basis of density functional theory, using the local spin density approximation (LSDA) for exchange and correlation in the parametrization supplied by Vosko et al. (1980).

The first step consisted of determining the self-consistent potentials, calculated within the framework of the spin-polarized relativistic screened Korringa-KohnRostoker (KKR) method (Szunyogh et al. 1994, Zeller et al. 1995) with the magnetic field pointing in each layer uniformly parallel to the (001) direction. No layer relaxation was taken into account, i.e. the crystalline structure corresponds to a fcc lattice with the lattice parameter of bulk copper. Further details are given by Újfalussy et al. (1996).

With the self-consistent potentials available, we represent the interface region by a finite cluster of potential wells. It was assumed that the sixth $\mathrm{Cu}$ layer counted inward starting from the $\mathrm{Fe} / \mathrm{Cu}$ interface is already identical to bulk copper. The same assumption was made for the other direction, i.e. towards the vacuum region. For all calculations a cluster of 12 shells containing 249 atoms has been used, and a reasonable convergence of the density of states (DOS) was achieved.

The corresponding APS spectrum for each Fe layer has been calculated with the non-relativistic approximation using the expressions derived by Ebert and Popescu (1997). The spin and layer-resolved APS signal $P_{\sigma}^{i}(E)$ is given by a weighted selfconvolution of the local DOS above the Fermi level:

$$
P_{\sigma}^{i}(E)=\int_{E_{\mathrm{F}}}^{E+E_{\mathrm{c}}-E_{\mathrm{F}}} \mathrm{d} E^{\prime} \sum_{l, l^{\prime \prime \sigma^{\prime \prime}}} n_{l \sigma \sigma}^{i}\left(E^{\prime}\right) n_{l, \sigma^{\prime \prime}}^{i}\left(E^{\prime \prime}\right) W_{l \sigma, l^{\prime \prime \sigma^{\prime \prime}}}\left(E^{\prime}, E^{\prime \prime}\right),
$$

where $E$ is the energy of the incoming electron, $E_{\mathrm{c}}$ the energy of the involved core level ( $2 \mathrm{p}_{3 / 2}$ in present calculations), $E_{\mathrm{F}}$ is the Fermi energy and the restriction $E-E^{\prime}=E^{\prime \prime}-E_{\mathrm{c}}$ applies due to the conservation of energy. Furthermore, $W_{l \sigma, l^{\prime}}\left(E, E^{\prime}\right)$ is an effective cross-section combining the various angular matrix elements as well as the radial Coulomb matrix elements and $n_{l \sigma}^{i}(E)$ is the angular momentum- and spin-resolved local DOS of layer $i$.

The total APS signal recorded in experiment represents a superposition of the individual layer signals, weighted to account for electron attenuation. Hence, the final expression for the total signal has the form:

$$
P_{\sigma}^{\mathrm{total}}(E)=\frac{1}{n} \sum_{i=1}^{n} \exp (-(i-1) d / \lambda) P_{\sigma}^{i}(E)
$$

with $d$ the layer thickness and $\lambda$ the electron mean free path length. The layer index $i$ runs inward to the interface starting from the top-most layer.

Finally, one should note that for a comparison with the experiment the results based on equation (1) have to be broadened to account for the various intrinsic and apparative broadening mechanisms as described by Ertl et al. (1993). In addition, the APS signal is recorded using the modulation technique which improves the signal-tonoise ratio and for this reason the energy derivative of $P_{\sigma}(E)$ is usually used. 


\section{§3. Results AND Discussion}

In our previous calculations for the ferromagnetic phase (Újfalussy et al. 1996) a strong charge depletion was found to characterize the Fe top-most layer. This feature, as well as the enhancement of the spin magnetic moments compared to those for buried Fe layers, is a general property of ferromagnetic surfaces and is attributed to the band narrowing at the surface (Ebert et al. 1998). Such behaviour is also reflected by the calculated APS spectra for the Fe top-most layer. The upper panel of figure 1 shows the APS signal of this layer (majority channel on the left, the minority on the right) for different numbers of Fe layers $n$ compared with the spectra for the Fe interface layer (lower panel). One should note that for $n=1$ the interface and top-most layers are identical. For all the other calculated systems $(n=2, \ldots, 6)$ the top-most layer related spectra present a narrower shape. Also the spin asym-

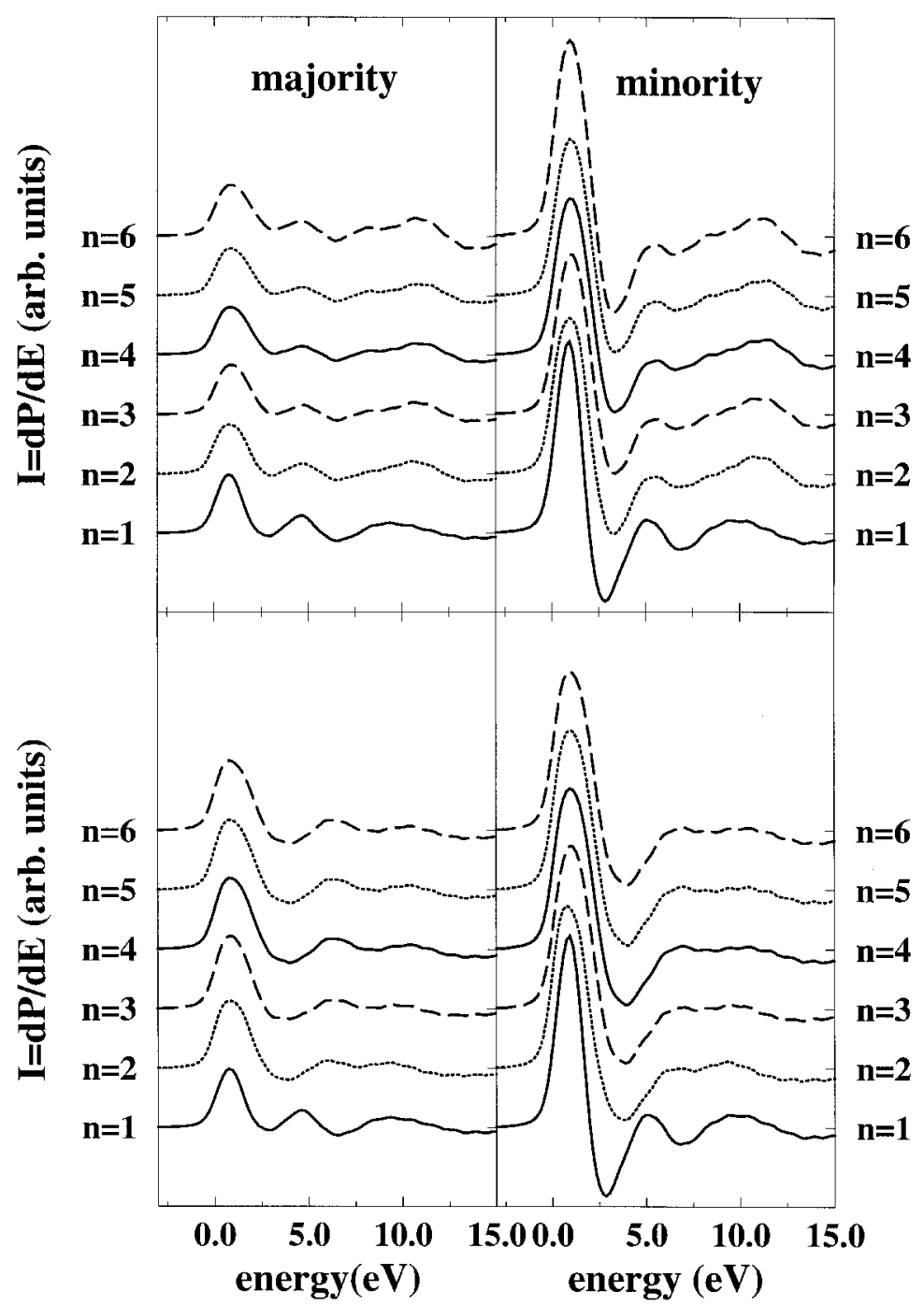

Figure 1. Spin-resolved APS spectra at the $2 \mathrm{p}_{3 / 2}$ edge for Fe top-most layer (upper panel) and $\mathrm{Fe}$ interface layer (lower panel) of the $\mathrm{Fe}_{n} / \mathrm{Cu}(001)$ overlayer system. 
metry, defined as $A=(I \uparrow-I \downarrow) /(I \uparrow+I \downarrow)$, is higher for these spectra, as a direct consequence of the corresponding stronger spin polarization (Újfalussy et al. 1996).

The total Fe-APS spectra computed according to equation (2) for an increasing number of Fe layers are plotted in the upper panel of figure 2. For both spin channels the main peak tends to become broader and the second positive peak moves towards higher energies and gets smoother. However, for $n \geq 4$, the changes are rather small indicating a kind of saturation. The spin asymmetry decreases as the number of $\mathrm{Fe}$ layers increases, although it is still too high, $45 \%$ for $n=6$, compared to the experimental one (Detzel et al. 1996) which is about $30 \%$. In order to investigate the origin of this discrepancy, layer-resolved APS spectra for $n=6$ are presented in the lower

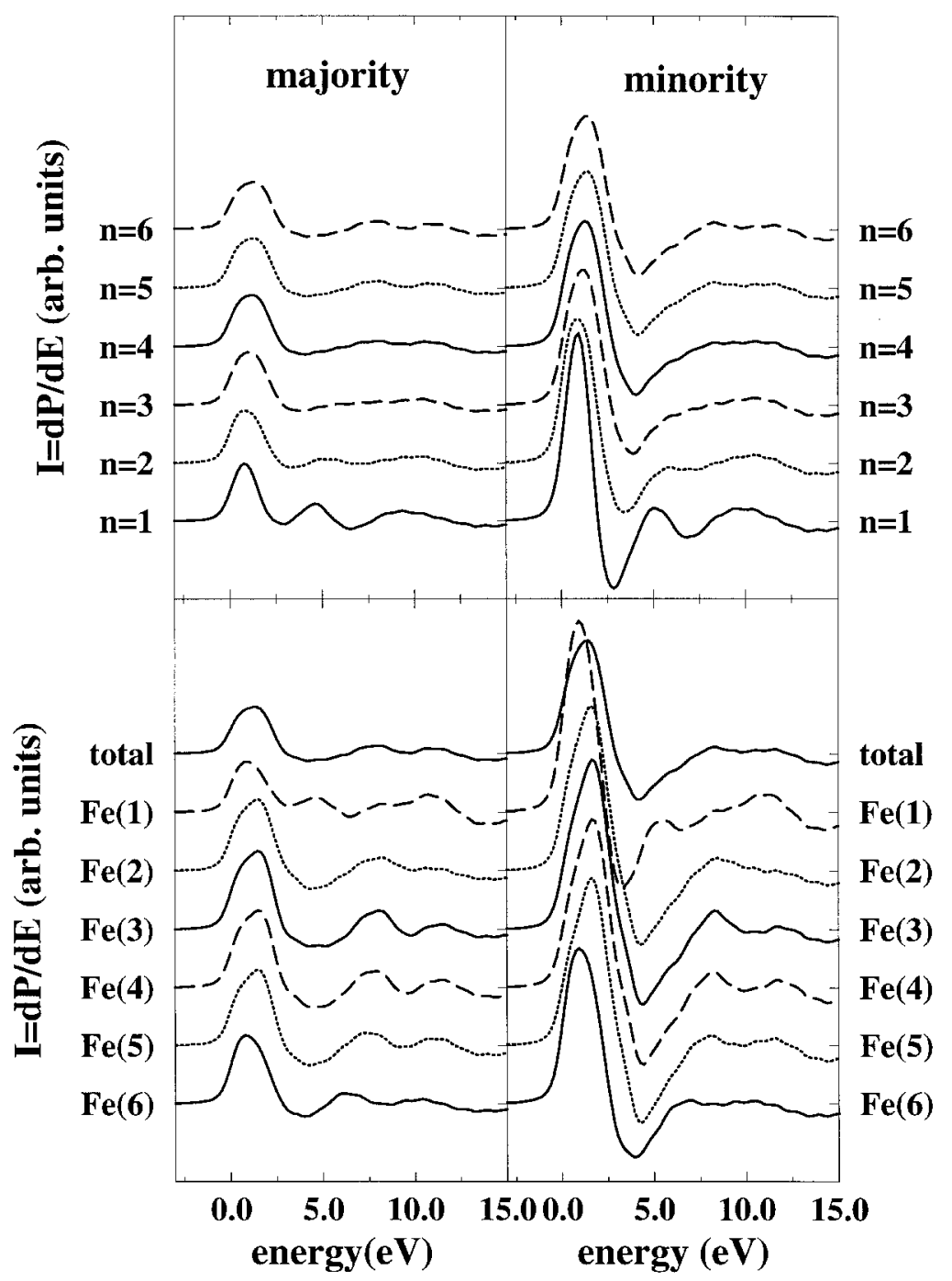

Figure 2. The total spin-resolved APS spectra at the $2 \mathrm{p}_{3 / 2}$ edge of $\mathrm{Fe}$ in $\mathrm{Fe}_{n} / \mathrm{Cu}(001)$, calculated using equation (2) (upper panel) and the total and layer-resolved spectra of $\mathrm{Fe}$ in $\mathrm{Fe}_{6} / \mathrm{Cu}(001)$ (lower panel). $\mathrm{Fe}(1)$ denotes the top-most layer and the counting runs inward to the interface. 
panel of figure 2. For a better comparison of their relative magnitude, the individual layer spectra are plotted without any weighting factor. The main peak of the total signal near the energy zero is strongly broadened because of the different position of this peak in each individual layer spectrum. One sees that the spectra for the inner layers $(i=2, \ldots, 5)$ exhibit the same features and clearly differ from those of the outer layers $(i=1,6)$. Compared to the latter ones, the band widths of the inner layers are larger and their spin polarization - reflected in the spin asymmetry of the spectra - is smaller. However, due to the slowly decreasing attenuation factor in equation (2), their role in the shape of the total signal is still important; namely, the spectra are close to a typical fcc-bulk behaviour and modulate the total signal.

The question of exaggerated predicted spin asymmetry still remains. The highmoment antiferromagnetic phase was recently investigated (Szunyogh et al. 1997) and certain antiferromagnetic configurations were found to be energetically more favourable. From the APS point of view, such a configuration would lead to a redistribution of the partial signals stemming from the two spin channels. As a result, a significant change in the spin asymmetry should occur. Investigations in this line that could open a better understanding of the magnetic properties of the system discussed are in progress.

\section{ACKNOWLEDGEMENTS}

This work was supported by the German Ministry of Education and Research (BMBF) within the programme Zirkular Polarisierte Synchrotronstrahlung: Dichroismus, Magnetismus und Spinorientierung and by the TMR-Network 'Interface Magnetism'. One of us (L.S.) also acknowledges the financial support from the Hungarian National Scientific Research Foundation (OTKA T024137). Financial support by the European Science Foundation (ESF) within the programme 'Relativistic Effects in Heavy Element Chemistry and Physics' during the early stages of the project is also gratefully acknowledged.

\section{REFERENCES}

Detzel, Th., Vonbank, M., Donath, M., and Dose, V., 1995, J. Magn. magn. Mater., 147, L1.

Detzel, Th., Vonbank, M., Donath, M., Memmel, N., and Dose, V., 1996, J. Magn. magn. Mater., 156, 287.

Ebert, H., and Popescu, V., 1997, Phys. Rev. B, 56, 10165.

Ebert, H., Popescu, V., Nonas, B., and Dederichs, P. H., 1998, Phys. Rev. B (submitted).

Ertl, K., Vonbank, M., Dose, V., and Noffke, J., 1993, Solid State Commun., 88, 557.

Li, D., Freitag, M., Pearson, J., Qiu, Z. Q., and Bader, S. D., 1994, Phys. Rev. Lett., 72, 3112.

Kirschner, J., 1984, Solid State Commun., 49, 39.

Reinmuth, J., Passek, F., Petrov, V. N., Donath, M., Popescu, V., and Ebert, H., 1997, Phys. Rev. B, 56, 12893.

Sz unyogh, L., Újfalussy, B., and Weinberger, P., 1997, Phys. Rev. B, 55, 14392.

Sz unyogh, L., Újfalussy, B., Weinberger, P., and Kollár, J., 1994, Phys. Rev. B, 49, 2721.

Thomassen, J., May, F., Feldmann, B., Wuttig, M., and Івасн, H., 1992, Phys. Rev. Lett., 69, 3831 .

Újfalussy, B., Szunyogh, L., and Weinberger, P., 1996, Phys. Rev. B, 54, 9883.

Vosko, S. H., Wilk, L., and Nusair, M., 1980, Can. J. Phys., 58, 1200.

Zeller, R., Dederichs, P. H., Újfalussy, B., Szunyogh, L., and Weinberger, P., 1995, Phys. Rev. B, 52, 8807. 